\title{
Musique et théorie queer
}

Music and Queer Theory

\section{(2) OpenEdition \\ Journals}

Édition électronique

URL : http://journals.openedition.org/transposition/414

DOI : 10.4000/transposition.414

ISSN : 2110-6134

Éditeur

CRAL - Centre de recherche sur les arts et le langage

Référence électronique

"Musique et théorie queer », Transposition [En ligne], 3 | 2013, mis en ligne le 01 mars 2013, consulté le 16 février 2020. URL : http://journals.openedition.org/transposition/414 ; DOI : 10.4000/ transposition. 414

Ce document a été généré automatiquement le 16 février 2020.

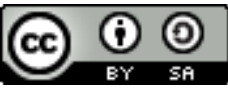

La revue Transposition est mise à disposition selon les termes de la Licence Creative Commons Attribution - Partage dans les Mêmes Conditions 4.0 International. 


\title{
Musique et théorie queer
}

\author{
Music and Queer Theory
}

[As the doctors drop the baby into an incubator,

the mother looks up.]

Patient : Is it a boy or a girl?

Obstetrician : Now, I think it's a little early to start imposing roles on it, don't you?

Monty Python's The Meaning of Life, Part I - The

Miracle of Birth, 1983.

1 Le mot «queer » intrigue. Il évoque dans l'imaginaire commun des mondes interlopes, sa sonorité même est mystérieuse. Pourtant, son emploi n'a cessé de s'étendre, et le terme renvoie aujourd'hui à un ensemble de phénomènes socio-culturels traversant les multiples strates de nos sociétés. Étonné-e par son succès et surtout par le fait qu'il en soit venu à représenter une forme de "chic culturel ", le-la philosophe et activiste queer Beatriz Preciado retrace l'histoire politique du terme. À l'origine, le terme «queer » était une insulte :

En anglais, depuis son apparition au XVIII e siècle, le mot « queer » servait à nommer

la personne ou la chose qui, parce qu'elle était inutile, difforme, fourbe ou excentrique, remettait en question le bon fonctionnement du jeu social. Étaient « queer » le tricheur, le voleur, l'ivrogne, la brebis galeuse [...] mais aussi tous ceux qui, de par leur particularité ou leur étrangeté, ne pouvaient pas être immédiatement reconnus comme homme ou comme femme ${ }^{1}$.

2 Ainsi, l'injure n'avait pas un contenu spécifique : elle semblait réunir en son sein tout ce qui était considéré comme détestable. Pour reprendre les mots de Preciado:

Le mot «queer» ne semblait pas définir une qualité de l'objet auquel il faisait référence. Il indiquait, par exemple, l'incapacité d'un locuteur à trouver une catégorie dans le domaine de la représentation qui puisse s'ajuster à la complexité de ce que le mot prétendait définir. Ainsi, dès le début, «queer » est davantage la marque d'une faille dans la représentation linguistique qu'un simple adjectif. [...] Ce qui, d'une certaine façon, équivaut à dire : ce que je nomme « queer » suppose qu'il $\mathrm{y}$ a un problème dans mon système de représentation; c'est une perturbation, une vibration étrange dans le champ de ma visibilité qui doit être caractérisée par l'injure ${ }^{2}$. 
Cette perturbation semblait venir surtout de tous les corps qui échappaient à l'institution hétérosexuelle et à ses normes; des corps qui, par leurs formes d'interaction et de production de plaisir, faisaient bouger les lignes de démarcation entre masculin et féminin, mais aussi entre organique et inorganique, animal et humain. Étaient considérés comme queer les déviants: homosexuels et lesbiennes, travestis ou sadomasochistes. Le mot servait à tracer une délimitation au sein de la sphère sociale : celui qui nommait l'autre queer se plaçait lui-même à l'intérieur de la sphère publique avec ses pairs hétérosexuels, tandis qu'il reléguait le queer aux confins de l'humain, hors de l'espace social, condamné au secret et à la honte. Cependant, comme le rappelle Preciado, "l'histoire politique d'une injure est aussi l'histoire changeante de ses usages, de ses usagers et de ses contextes d'énonciation ${ }^{3}$. Il se trouve justement qu'en moins de deux siècles, l'injure " queer » a changé d'usages, d'usagers et de contextes d'utilisation.

4 Au milieu des années 1980, les activistes de groupes féministes radicaux comme Lesbian Avengers ou Radical Furies décidèrent de se réapproprier l'injure « queer » pour définir leur terrain d'action : critique sociale et politique, intervention dans le champ culturel et résistance à la normalisation. Le sujet d'énonciation se trouve alors changé : ce n'est plus l'hétérosexuel qui traite l'autre de «pédé » ou de « gouine », mais l'homosexuel, la lesbienne ou le transsexuel qui s'auto-dénomme queer, opérant une rupture intentionnelle avec la norme ${ }^{4}$. Il s'agit également d'affirmer le caractère politique (pour ne pas dire policier) de la construction de notions comme celles d'homosexualité et d'hétérosexualité, puis de remettre en question leur légitimité à délimiter le champ social. Ainsi, dans nos sociétés contemporaines, le mot "queer " a cessé d'être une injure et un instrument de répression sociale pour devenir un signe et un moyen de résistance à la normalisation.

Dans le domaine universitaire, un projet critique, héritier de la tradition féministe et anticoloniale, donna lieu à la constitution d'un corpus de textes signés par Teresa de Lauretis, Judith Butler, Eve Kosofsky Sedgwick ou Michael Wargner, pour ne citer que quelques-un.e.s des auteur.e.s. On commença peu à peu à se référer à ces textes en invoquant l'expression de "théorie queer $»^{5}$. Fortement influencée par les travaux de Michel Foucault (son Histoire de la sexualité et des concepts, celui de «biopolitique » en particulier) et le déconstructionnisme derridien, cette théorie se fondait en partie sans que cela constitue une tendance homogène - sur l'analyse et la déconstruction des processus historiques et culturels ayant conduit à l'invention du corps hétérosexuel blanc comme norme. Cette norme était désormais présentée comme une fiction occidentale dominante qui avait mené à l'exclusion des différences hors du champ de la représentation politique ${ }^{6}$. Comme l'affirme Beatriz Preciado :

Les élaborations de la théorie « queer » réalisées durant les années 1990 par Judith Butler ou par Eve K. Sedgwick ont révélé que les expressions, apparemment descriptives, «c'est une fille » ou "c'est un garçon », prononcées au moment de la naissance (ou même au moment de la visualisation du foetus lors de l'échographie) ne sont que des invocations performatives - plus proches des expressions contractuelles prononcées lors des rituels sociaux comme le « oui, je le veux » du mariage, que des énoncés descriptifs tels que « ce corps a deux jambes, deux bras et une queue ». Ces injonctions performatives sur le genre sont des éléments de langage chargés historiquement du pouvoir d'assigner au corps une identité sexuelle - masculin ou féminin - ainsi que de sanctionner les corps qui menacent la cohérence du système sexe/genre jusqu'au point de les soumettre à des processus chirurgicaux de "cosmétique sexuelle» (diminution de la taille du clitoris, 
augmentation du pénis, fabrication de seins en silicone, reféminisation hormonale du visage, etc. $)^{7}$. considérée comme une construction sociale, historique et culturelle qui ne serait pas déterminée par une vérité ou un substrat naturel ou ontologique. Il donna naissance à une réflexion sur les modes de subjectivité et d'identité, ainsi que sur la construction sociale et politique du sexe ${ }^{8}$.

7 Le domaine de l'esthétique n'a pas échappé à cette grille de lecture, utilisée notamment pour analyser la performativité des genres et les processus de production corporels de la masculinité et la féminité. L'introduction de ces nouvelles approches a favorisé l'émergence d'études inédites, à travers lesquelles se joue un double apport disciplinaire : l'historiographie artistique traditionnelle s'est vue enrichie par les outils issus de la théorie queer, tandis que les études queer intégraient peu à peu des objets nouveaux à leur champ d'investigation?.

8 La réflexion portant sur l'objet « musique » n'est pas restée à l'écart, donnant lieu à la création de groupes de recherche et à la publication d'ouvrages, d'articles et de thèses abordant des thématiques jusqu'alors relativement peu traitées, voire taboues, ayant souvent trait à la construction des identités sexuelles (voir à ce propos les passages relatifs à la New Musicology et au gender studies dans l'entretien avec Annegret Fauser publié dans ce numéro) ${ }^{10}$. D'un point de vue méthodologique, on pourrait affirmer que cette perspective a stimulé un renouvellement théorique récemment intervenu à l'échelle internationale. Les méthodes «traditionnelles » issues de l'analyse musicale et de la musicologie historique se sont ouvertes à l'histoire, à la sociologie, à la littérature, à l'esthétique ou encore à l'anthropologie afin de mieux saisir le processus de construction de l'objet « musique » et sa dimension sociale dans toute leur complexité. Ce numéro de Transposition. Musique et sciences sociales entend ainsi participer à ce renouveau disciplinaire en publiant dans ce dossier thématique des articles et des études de cas s'appuyant sur les théories queer - encore peu mobilisées en France pour analyser différents champs et aspects du monde musical : ceux de la création, de l'écoute, de la typologie vocale et de la représentation musicale des genres.

«Musicality, Essentialism, and the Closet», paru dans le volume désormais classique intitulé Queering the Pitch. The New Gay and Lesbian Musicology, et co-édité en 1994 par Elizabeth Wood, Gary C. Thomas et Brett lui-même. Spécialiste de l'œuvre de William Byrd et de Benjamin Britten, Brett fut l'un des précurseurs aux États-Unis des études musicologiques gays et lesbiennes. Il participa, entre autres, à la création en 1989 de la Gay and Lesbian Study Group au sein de l'American Musicological Society ${ }^{11}$. Dans cet article, fortement influencé par les travaux de Mary McIntosh, de David Halperin et surtout d'Eve Kosofsky Sedgwick (Epistemology of the Closet), Brett compare le terme d' « homosexualité » à celui de " musicalité », en ce qu'ils peuvent renvoyer l'un comme l'autre à la figure du "déviant» dans la culture occidentale moderne. Après avoir exposé les discours de penseurs qui, depuis Platon et Aristote jusqu'aux psychanalystes post-lacaniens français, ont pris leurs distances avec la musique sous le prétexte de son ambiguïté morale - la musique risquant alors d'entraîner ceux qui la pratiquent sur le chemin de la déviance -, Brett rappelle les diverses tentatives effectuées pour maîtriser ce danger à travers les âges. Il commente ainsi les efforts fournis par l'académisme pour affirmer la virilité dans le domaine de la musique. Des phénomènes aussi divers 
que la rhétorique de la transcendance de la musique absolue, la mise en avant des compétences purement techniques dans l'enseignement musical, la promotion d'un canon exclusivement allemand ou la «mise au placard» de l'homosexualité de compositeurs comme Franz Schubert ou Britten, sont considérés par Brett comme autant de démarches visant à lier la musique au pouvoir et à la hiérarchie, afin de la préserver de toute forme de déviance. Rédigé à l'époque où la New Musicology était en train de se forger, cet essai très représentatif du courant invite à une prise de conscience: Brett tente de mettre en évidence les traits qui ont façonné de manière plus ou moins visible les institutions musicales, non seulement à travers les programmes d'études et les frontières disciplinaires, mais aussi en déterminant ce que doit être la «bonne musique » et même ce qui peut être défini comme étant de la musique.

L'article de Fred Everett Maus "Classical Concert Music and Queer Listening " prend d'ailleurs comme point de départ deux textes issus de la New Musicology étasunienne, Feminine Endings: Music, Gender, and Sexuality ${ }^{12}$ de Susan McClary et l'article de Suzanne G. Cusick «On a Lesbian Relationship with Music: A Serious Effort Not to Think Straight $»^{13}$, qui ont ouvert des perspectives sur les rapports entre musique et sexualité. Maus s'appuie sur la discontinuité entre expérience et comportement dans l'écoute du concert classique - phénomènes que McClary et Cusick avaient assimilés à de puissantes oppositions culturelles entre inner et outer, public et privé - afin de déterminer quels modèles d'écoute pourraient dériver des études sur la subjectivité sexuelle et offrir des éléments d'analyse alternatifs pour aborder l'écoute passive ou active.

11 Les rapports que l'on peut établir entre musique et sexualité sont également au cœur de l'article d'Esperanza Miyake «Understanding Music and Sexuality through Ethnography : Dialogues between Queer Studies and Music ». À partir des théories queer sur la musique, Miyake explore la manière dont pourraient être conceptualisés les rapports entre musique et sexualité. Après avoir observé comment les questions de désir et de plaisir entrent en jeu dans une telle relation et interrogé celle-ci à l'aide de termes queer, queerness et queering, Miyake soutient que nous devons construire d'autres modes de pensée pour l'aborder, modes de pensée qui comprennent mais ne se résument pas aux questions de genre et d'érotisme. D'un point de vue méthodologique, Miyake privilégie la recherche ethnographique et invite les chercheurs à utiliser cette forme de collecte de données empiriques, ainsi que les outils théoriques qu'elle mobilise, pour mener des études inspirées par la théorie queer dans le domaine de la musique.

12 C'est ce que proposent les deux textes suivants, fondés sur une approche ethnographique. L'article de Maria Katharina Wiedlack «"I don't give a shit where I spit my phlegm" (Tribe 8). Rejection and Anger in Queer-Feminist Punk Rock » explore le punk queer-féministe à travers quelques exemples de performers punk rock (Tribe 8, Agatha, Stag Bitten) et leurs productions artistiques. Par l'analyse de leur musique et la mobilisation de notions comme le rejet et la colère, Wiedlack montre que ces groupes utilisent le terme queer en tant que marque anti-identitaire et anti-sociale. Cette interprétation est influencée par la théorie queer anti-relationnelle élaborée par des auteurs comme Lee Edelman, qui définissent le queer comme une force négative dans la sexualité, contrariant la cohérence psychique du sujet et empêchant par conséquent l'établissement de relations sociales. Pour ces activistes punk queer-féministes, se 
définir ainsi serait un moyen de résister à la misogynie de la culture mainstream ainsi qu'à celle des milieux de la contre-culture.

De son côté, dans «Boy Bands, Drag Kings, and the Performance of (Queer) Masculinities", Jennifer Moos examine les potentialités queer de la culture des boy bands. S'appuyant sur les textes de Judith Butler, Judith «Jack » Halberstam, et Gayle Wald, Moos propose une analyse de ces groupes, en prenant comme exemples les Backstreet Boys ou Take That, et conteste leur appartenance à l'hétéronormativité. Selon l'auteur, la culture des boy bands offre un espace au déploiement de masculinités (queer) alternatives. Pour le démontrer, Moos intègre à son étude les réinterprétations subculturelles de la masculinité des boy bands pratiquées par les troupes de drag kings qui utilisent des stratégies d'exagération et de parodie pour déconstruire de façon ludique les notions d'identité de genre binaire.

14 L'article de Raphaëlle Legrand «Orphée baro/queer » s'attache à déconstruire le mythe d'Orphée à travers l'histoire de ses multiples réactualisations lyriques, de Jacopo Peri à Christoph Willibald Gluck. Après avoir montré comment les aspects les plus violents du mythe sont partiellement masqués afin de les faire entrer dans le cadre d'un spectacle lyrique, Legrand étudie l'érotisme propre au personnage d'Orphée (inventeur de l'homosexualité d'après Ovide) et la misogynie croissante témoignée à l'égard du personnage d'Eurydice. La seconde partie propose une interprétation de l'Orphée de Gluck, de la pluralité des tessitures utilisées (soprano, alto, ténor, baryton), variables selon les époques, et de l'idéal du "chanteur par excellence». Elle s'intéresse également à la tradition du travesti initiée par Pauline Viardot, qui représente un réinvestissement du personnage d'Orphée, et enfin à la rivalité mezzo/contre-ténor qui caractérise l'époque contemporaine.

La question de la vocalité et du genre est au centre de l'article pionnier d'Alexandros $\mathrm{N}$. Constansis «The Female-to-Male (FTM) Singing Voice and its Interaction with Queer Theory : Roles and Interdependency ». S'appuyant sur l'endocrinologie, sur des sources médicales et sur deux études de cas, Constansis étudie en détail les possibilités vocales de « celles » qui, dans un processus de réassignation sexuelle, deviendront des hommes. Comme l'affirme l'auteur, connaître davantage les possibilités sonores de la «transvocalité » aiderait sans doute à s'affranchir du modèle binaire. La trans-vocalité pourrait alors devenir une forme de vocalité parmi d'autres.

Le mythe d'Orphée est à nouveau abordé dans l'étude de cas d'Oliver C. E. Smith « "The Cult of the Diva" - Rufus Wainwright as Opera Queen ». L'article analyse la manière selon laquelle le chanteur explore l'(homo)sexualité dans ses productions musicales en s'appropriant la figure d'Orphée. Pour Smith, c'est un moyen utilisé par Wainwright pour réinterpréter l'histoire culturelle, et réviser le stéréotype péjoratif qu'est l'opera queen.

17 La publication d'un tel dossier revêt pour le comité de rédaction de Transposition. Musique et sciences sociales une importance scientifique particulière : il semble en effet que les études sur le genre dans le domaine de la musique, en France, se soient pour le moment presque exclusivement intéressées à la question de la place des femmes dans l'histoire de la musique, pour dénoncer leur oubli systématique et mettre en lumière les présupposés « virils» et souvent misogynes de la construction de cette histoire. Il ne s'agit nullement pour nous de contester l'intérêt épistémologique et social d'une telle approche de la question du genre, à qui l'on souhaite de s'établir durablement dans le paysage de la recherche française et internationale. Cependant, cette entrée dans la 
question est une entrée possible parmi d'autres, puisque le genre se décline de façons multiples et non pas uniquement sous la forme de l'opposition masculin / féminin. Les théories du genre ont montré au cours des dernières décennies que les catégories d'« homme » et de «femme " ne sont pas les seules catégories valables de l'analyse, et que cette construction binaire et exclusive de toute autre forme d'identité sexuelle a elle-même une histoire que l'on peut retracer.

Les luttes sociales et politiques des groupes «minoritaires » ont abouti dans le courant $\mathrm{du} \mathrm{XX}^{\mathrm{e}}$ siècle à un élargissement considérable de la notion de genre, permettant en retour de questionner les diverses constructions de genre de manière plus ouverte. Le terme "queer" renvoie sans doute plus que celui de "genre " à cette ouverture radicale, ce qui, à notre avis, transparaît dans les diverses thématiques abordées dans les articles que nous présentons ici (qui s'intéressent, sur un mode pragmatique, aux identités homosexuelles, transsexuelles, ainsi qu'aux formes alternatives de masculinité et de féminité). Notons également, par ailleurs, que ces articles portent pratiquement tous sur des sujets plus ou moins contemporains, ce qui semblerait signifier que le champ possible d'application de la catégorie de "queer» correspond davantage à l'époque contemporaine. Cette idée doit néanmoins être nuancée à la lecture de l'article de Raphaëlle Legrand, pour prendre un exemple, qui s'intéresse aux représentations opératiques du mythe d'Orphée à travers les siècles, et qui démontre de manière exemplaire que la théorie queer peut finalement s'appliquer à toutes les époques. Il s'agit donc désormais de continuer à défricher le terrain ouvert par la théorie queer.

Par le choix de la thématique de ce numéro, nous avons ainsi souhaité contribuer à la diffusion d'un courant de pensée, celui des queer studies, qui ne s'est pour le moment pas vraiment établi ni développé dans le cadre des études françaises sur la musique (notons par exemple que sur les douze propositions d'articles reçues pour ce numéro, seules deux étaient en langue française). Alors qu'il semble aujourd'hui essentiel d'engager une réflexion pour penser les politiques et poétiques du genre - notamment au sein de la musicologie française -, le recours à la théorie queer, déjà bien ancrée dans les pays anglo-saxons, semble indispensable.

Nous voudrions conclure cet éditorial en remerciant chaleureusement les lecteurs et lectrices externes qui ont participé à ce dossier : Marie-Hélène Bourcier, Susan Campos Fonseca, Lisa Colton, Freya Jarman, Kyrre Tromm Lindvig, Beatriz Preciado et Jodie Taylor.

\section{NOTES}

1. PRECIADO, Beatriz, "Queer: historia de una palabra", in Parole de Queer 1, en ligne : http:// paroledequeer.blogspot.com.es/2012/04/queer-historia-de-una-palabra-por.html. Nous sommes responsables des traductions françaises. Le début de notre éditorial est largement inspiré du texte de Preciado. Docteur en philosophie et disciple de Jacques Derrida et d'Agnès Heller, Preciado explore, à partir d'une définition biopolitique du corps, la production du genre, du sexe 
et de la sexualité, ces entités étant envisagées comme des techniques spécifiques de gouvernement des corps. Voir : PRECIADO, Beatriz, Manifeste contra-sexuel, Paris, Balland, 2000 ; id., Testo Junkie. Sexe, drogues et biopolitique, Paris, Grasset, 2008 ; id., Pornotopie. Playboy et l'invention de la sexualité multimédia, Paris, Flammarion, 2011.

2. Ibid.

3. Ibid.

4. Cette absence de catégorie spécifique d'individu associée au terme " queer " constituera l'un de ses signes distinctifs. Dans son livre Saint Foucault. Towards a Gay Hagiography, David Halperin affirme ainsi : "Queer is by definition whatever is at odds with the normal, the legitimate, the dominant. There is nothing in particular to which it necessarily refers. It is an identity without an essence. "Queer" then, demarcates not a positivity but a positionality vis-à-vis the normative [...] [Queer] describes a horizon of possibility whose precise extent and heterogeneous scope cannot in principle be delimited in advance. » HALPERIN, David, Saint Foucault: Towards a Gay Hagiography, New York, Oxford University Press, 1995, p. 62.

5. Teresa de Lauretis fut la première à utiliser l'expression "théorie queer». DE LAURETIS, Teresa, "Queer Theory, Lesbian and Gay Studies : An Introduction», in differences : A Journal of Feminist Cultural Studies, vol.3, № 2 (special issue), summer, 1991, p. iii-xviii. Pour une introduction à la théorie queer voir: TURNER, William B., A Genealogy of Queer Theory, Philadelphia, Temple University Press, 2000 ou SULLIVAN, Nikki, A Critical Introduction to Queer Theory, New York, New York University Press, 2003.

6. Voir par exemple : BUTLER, Judith, Gender Trouble. Feminism and the Subversion of Identity [1990], New York, London, Routledge, 1999; SEDGWICK, Eve Kosofsky Epistemology of the Closet [1990], Berkeley, University of California Press, 2008; WARNER, Michael, Fear of a Queer Planet. Queer Politics and Social Theory, Minneapolis, University of Minnesota Press, 1994.

7. PRECIADO, Beatriz, Manifiesto contra-sexual, Barcelona, Anagrama, 2011, p. 20. Nous sommes responsables de la traduction française.

8. Pour ce qui est du contexte français, il faut citer les travaux précurseurs engagés par Didier Eribon, en particulier le colloque qu'il organisa en 1997 sur les études gay, lesbiennes et la théorie queer au centre Pompidou, auquel participèrent Monique Witting et Eve Kosofsky Sedgwick (cf. Les Études gay et lesbiennes, Paris, Centre Pompidou, 1998) et le séminaire qu'il consacra à ce même sujet à l'Ehess entre 1998 et 2004, où furent invités des chercheurs tant américains que français. Voir aussi les travaux de Marie-Hélène Bourcier: Queer Zones [2001], Paris, Editions Amsterdam, 2006 et Sexpolitiques : Queer Zones 2, Paris, La Fabrique, 2005.

9. Voir par exemple les travaux de María Mercedes Liska sur le tango queer. LISKA, María Mercedes, "El tango como disciplinador de cuerpos ilegítimos-legitimados ", in Trans. Revista Transcultural de Música, № 13, 2009, en ligne: http://www.sibetrans.com/trans/a53/el-tangocomo-disciplinador-de-cuerpos-ilegitimos-legitimados.

10. Voir par exemple : KOESTENBAUM, Wayne, The Queen's Throat. Opera, Homosexuality, and the Mystery of Desire, New York, Poseidon Press, 1993 ; BRETT, Philipp, WOOD, Elizabeth, THOMAS, Gary C. (eds.), Queering the Pitch. The New Gay and Lesbian Musicology [1994], New York, London, Routledge, 2006; SOLIE, Ruth A. (ed.), Musicology and Difference: Gender and Sexuality in Music Scholarship, Berkeley, Los Angeles, London, University of California Press, 1995 ; FULLER, Sophie et WHITESELL, Lloyd (eds.), Queer Episodes in Music and Modern Identity, Urbana, University of Illinois Press, 2002 ; HUBBS, Nadine, The Queer Composition of American's Sound, Berkeley, Los Angeles, London, University of California Press, 2004 ; PERAINO, Judith A., Listening to the Sirens. Musical Technologies of Queer Identity from Homer to Hedwig, Berkeley, Los Angeles, London, University of California Press, 2006 ; WHITELEY, Sheila et RYCENGA, Jennifer (eds.), Queering the Popular Pitch, New York, London, Routledge, 2006 TONGSON, Karen, Relocations: Queer Suburban Imaginaries, New York, New York University Press, 2011 ; JARMAN-IVENS, Freya, Queer Voices: Technologies, Vocalities, and the Musical Flaw, New York, Palgrave Macmillan, 2011 ; TAYLOR, Jodie, 
Playing it Queer: Popular Music, Identity and Queer World-making, Bern, Berlin, Bruxelles, Frankfurt am Main, New York, Oxford, Wien, Peter Lang, 2012.

11. Voir BRETT, Philip (compiled by), Benjamin Britten, Peter Grimes, New York, Cambridge University Press, 1983 et id., Music and Sexuality in Britten. Selected Essays, Berkeley, Los Angeles, London, University of California Press, 2006. Après son décès en 2002 a été décidée la remise d'un prix annuel, le «Philip Brett Award " de l'American Musicological Society, qui prime les études musicologiques réalisées dans le domaine des études gays, lesbiennes, bisexuelles et transgenres/ transsexuelles.

12. MCCLARY, Susan, Feminine Endings : Music, Gender, and Sexuality [1991], Minneapolis, University of Minnesota Press, 2002. Pour un bilan de l'auteure concernant les vingt ans qui se sont écoulés après la première publication du volume, voir McCLARY, Susan, «Feminine Endings at Twenty ", in Trans. Revista Transcultural de Música, № 15, Dossier : Música y estudios sobre las mujeres / Music and Women's Studies, editores invitados / guest editors : CAMPOS FONSECA, Susan et LORENZO ARRIBAS, Josemi, 2011, en ligne: http://www.sibetrans.com/trans/articulo/348/ feminine-endings-at-twenty.

13. CUSICK, Suzanne G., «On a Lesbian Relationship with Music : A Serious Effort Not to Think Straight », in BRETT, Philip, WOOD, Elizabeth et THOMAS, Gary C. (eds.), op. cit., p. 67-83. 